\title{
Capturing the Experiences of ESL Graduate Students in Engineering Educa- tion
}

\section{Ms. Hoda Ehsan, Purdue University-Main Campus, West Lafayette (College of Engineering)}

Hoda is a Ph.D. student in the School of Engineering Education, Purdue. She received her B.S. in mechanical engineering in Iran, and obtained her M.S. in Childhood Education and New York teaching certification from City College of New York (CUNY-CCNY). She is now a graduate research assistant on $\mathrm{STEM}+\mathrm{C}$ project. Her research interests include designing informal setting for engineering learning, and promoting engineering thinking in differently abled students in informal and formal settings.

Matilde Luz Sanchez-Pena, Purdue University-Main Campus, West Lafayette (College of Engineering)

Matilde Sanchez-Pena is a Visiting Assistant Professor of Engineering Education at Purdue University. She completed her Ph.D. at the same institution in 2018. Her dissertation explored differences across gender of faculty retention and promotion at research-intensive institutions. Dr. Sanchez-Pena aims to promote a more equitable engineering field, in which students of all backgrounds can acquire the knowledge and skills to achieve their goals. Before engaging in Engineering Education research, she completed graduate degrees in Industrial Engineering and Statistics and contributed to a wide range of research areas including genetic disorders, manufacturing optimization, cancer biomarker detection, and the evaluation of social programs.

Mr. Hossein Ebrahiminejad, Purdue University-Main Campus, West Lafayette (College of Engineering)

Hossein Ebrahiminejad is a Ph.D. student in Engineering Education at Purdue University. He completed his M.S. in Biomedical Engineering at New Jersey Institute of Technology (NJIT), and his B.S. in Mechanical Engineering in Iran. His research interests include student pathways, educational policy, and quantitative research methods.

\section{Mr. Hassan Ali Al Yagoub, Purdue University-Main Campus, West Lafayette (College of Engineering)}

Hassan Al Yagoub is a Ph.D. student in Engineering Education at Purdue University. His research interests include diversity \& inclusion, students' persistence, advising and mentoring, engineering career pathways, and school-to-work transition of new engineers. He holds a B.S. in Mechanical Engineering from University of Wisconsin-Milwaukee and a M.S. in Mechanical Engineering from Georgia Institute of Technology. Prior to beginning his doctoral studies, Hassan worked for five years at General Electric where he graduated from their Edison Engineering Development Program (EEDP) and then worked as a gas turbine fleet management engineer. In addition to his technical role, Hassan supported the recruiting, interview, and selection process of the EEDP Program, where he mentored interns, co-ops and Edison associates from the Middle East and Africa regions by developing and teaching a technical training curriculum, providing guidance for graduate school applications, and providing career consultation. 


\title{
Capturing the Experiences of ESL Graduate Students in Engineering Education
}

\begin{abstract}
Doctoral education can be a challenging and overwhelming journey for many graduate students. Engineering Education as an interdisciplinary and emerging field welcomes diverse students in terms of race and ethnicity, gender, nationality, sexual orientations, abilities, and language. Given the diversity within this field, engineering education students' experiences in this journey can be very different from one another during their doctoral years. Like any other diverse settings, engineering education students may have needs in common or completely different which required different ways of support.

In this study, we are a group of engineering education students and alumni who speak English as our second language (ESL). Using co-operative inquiry, we aimed to reflect on our doctoral journey in engineering education and highlight the challenges we went through and ways we were able to overcome them. We are taking the positionality of researcher to participant to examine our experiences. The challenges are mostly centered over cultural differences, identity formation, language barriers and expectations from ourselves and our community including peers, faculty, staff, and people beyond the engineering education community.

This study can convey a message to other ESL graduate students that they are not alone in this journey. Furthermore, the findings of our study highlights the approaches that other ESL students can take to help them overcome the challenges that they may face. Finally, the findings of this study can inform current and future faculty, policymakers, and school administrators about the ways they can support their ESL students.
\end{abstract}

\section{Introduction}

The reasons to pursue graduate education vary among individuals. Nevertheless, many of the challenges that are present during graduate training are common among graduate students. These similarities are more evident among graduate students that share certain backgrounds, and in particular among members of minority groups. While much research has been devoted to the experiences of gender and ethnic minorities [1]-[3], the experiences of English as Second Language (ESL) students in graduate school has been poorly explored. The challenges of ESL students often interplay with those of gender and ethnic minorities because their identities are intersectional with such characteristics. Therefore, being an ESL student represents a dimension that would be valuable to explore in the context of graduate education.

Supporting the development of graduate students have significant implications for graduate programs. Estimates of dropouts from graduate programs reach up to $50 \%$ at some institutions 
and programs [4]. The field of engineering is among the highest cumulative completion rates, with about $56 \%$ of students enrolled completing a Ph.D. degree within seven years [5]. In addition, further challenges experienced during graduate school might make it more difficult for students to persist. A recent survey found that graduate students were six times more likely to experience depression and anxiety than people in the general population [6], some of the identified reasons behind such estimates were being a gender minority, experiencing lack of work-life balance, and poor quality of the mentorship experience with academic advisors. While these issues have been explored in the general population of graduate students, there is no evidence of how these layered challenges accumulate for ESL students.

In the case of STEM-specific fields, a similar list of reasons has been identified for the attrition of graduate students. In their review of the literature, Satterfield et al. [7] identified three levels of the experiences of graduate students in STEM that impacted their attrition: 1) the individual level, i.e. advising relationships; 2) the programmatic, i.e. forms of funding support and professional development, such as teaching assistantships; and 3) the external, i.e. family. Despite this evidence, few is known about these challenges and how to target them systematically within programs. Therefore, it remains to the graduate students to create knowledge to support each other during their journeys through graduate education. Thus, this study takes the approach of researcher to participant, to uncover our own experiences as ESL graduate students.

The experiences we have during our doctoral training influence the development of an identity as researchers and as potential future scholars that will facilitate the education of the new generations or will perform successfully in non-academic contexts. The construction of such identities would influence our persistence in our graduate programs. A recent study about engineering doctoral students used Identity-Based Motivation theory to explore how graduate students experienced fit within their programs and how such fit motivated them to persist towards their goal of obtaining a Ph.D. Their results identified two general themes: a) graduate students developed their identity congruence by relating their research to real societal impact, in this sense, students found that their graduate degree was worth pursuing because they were having a real positive impact in the existing body of knowledge that would benefit the world; and b) the level of autonomy perceived from advisor and lab groups influenced their perceived identity congruence, with students with low autonomy feeling more detached and disappointed by their graduate experiences [8]. Further analyses of the different factors that play a role in the identity development of graduate students would help advance the understanding of how to better support their progress towards completion of their degrees.

\section{Purpose of the Study}

Graduate degrees in engineering education have a variety of characteristics that make them different from traditional engineering graduate degrees. As a consequence, socialization can take an even more critical role in the identity development of $\mathrm{Ph}$.Ds in this field. This process has 
been referred to as a journey or transformative experience. Aelong et al. [9] explored their personal and collective experiences of growth during their time in a PhD. in engineering education program. With a similar scope, in this study, the overarching research question that we seek to explore is: Which elements shape the experiences of ESL PhD students in an engineering education program?

We are aiming to unpack our individual and collective challenges as ESL graduate students in an engineering education program in the United States. Following a co-operative inquiry, we try to uncover elements that could support other generations of ESL students in the field and support their persistence and success through their graduate education path.

\section{Methods}

In this study, we utilize co-operative inquiry as our methodology to make meaning of shared experiences as Ph.D. students in Engineering Education discipline. Co-operative inquiry was first developed in 1986, as Heron started reflecting on the mutual gazing in interpersonal encounters. According to him, this mode of investigation involves two or more people researching one topic they are experiencing [10]. Researchers undergo a cycle of two phases: (1) experiencing and (2) reflecting. Each person is co-subject in the experience phases and then co-researcher in the reflection phases. All subjects (who are also researchers) are fully involved in all research decisions from content to methods.

This mode of inquiry has four main inquiry outcomes including, transformational, presentational, propositional, practical. Depending on the project, these outcomes are not mutually exclusive, and outcomes of the project can have aspects of each category. In this study, while we see how reflecting of our experiences helped with our transformations, we position this paper as a propositional report which is informative about the inquiry domain (i.e. what we are exploring) describe the inquiry method and provide a report on our findings. We also believe that the outcome of this study can lead to practical actions of transformations in the broader engineering education community including students, instructors, and administrators.

\section{Co-Subjects: Co-Researchers}

As previously mentioned, the authors of this paper are all the co-subjects and coresearchers of this study. Therefore, data of this study is produced by us (the researchers) as we reflect, share and discuss our experiences of being ESL students in an engineering education program. Two of us are second and third-year doctoral students respectively, one is a doctoral candidate in engineering education, and one recently graduated from the same discipline and is now an engineering faculty. In the journey of being graduate students in engineering education, we have a lot in common, but also many differences. All four of us identify English to be our second language; however, the level of comfort with English differs among us. Only two of us share a first language. While we identify as engineering education researchers, we are from different research groups and have varied research interests. We have all taken the same foundational courses in engineering education, mostly with the same instructors. 
This inquiry is internally initiated as all of us have been internal to the inquiry domain of being ESL engineering education students [10]. Therefore, we are members of the inquiry and are viewing this research from our internal perspectives. In this study, we are reflecting on our experience in relation to the environment and people around us (i.e. peers, staff, instructors and advisors).

\section{Launching an Inquiry Group}

The first step in this inquiry mode is to form an inquiry group. Heron [10] has introduced three main ways to launch an inquiry group. Two of them involve researchers' initiation and one happens within a pre-formed group. This group was formed by an initiator's call who is the second author of this paper. Consistent with what Heron describes, the initiator had read and reflected on the co-operative inquiry method before and was keen to explore the inquiry domain: experiences of ESL students in the engineering education discipline. Then, the initiator researcher called for collaborators who were likely to share the keenness of the topic.

After the collaborators responded to the initiator's call, the initiator talked to each individual separately and discussed the goal in mind and asked for their insights and feedback. The first meeting was then scheduled and all the researchers participated. We talked about the individual's interest in this topic, the detail of what this co-operative inquiry is in terms of defining the phases of experiencing and reflecting and the procedures for conducting the phases. Through a brainstorming session, we decided on the sub-topics we wanted to reflect on, the structure of phases and how often we wanted to meet to discuss our reflection and reflect on our observations.

\section{Data Collection and Inquiry Procedure}

During the first meeting, we decided on two main topics to explore: (1) Culture and (2) Language. Then we created a list of sub-topics for each category but agreed not to limit ourselves to those sub-topics when reflecting but using them only as a guide. With the list of topics and subtopics as guidelines, each of the authors wrote individual reflections which were later shared verbally in biweekly sessions. During the sessions, as individuals were sharing their reflections, others were allowed to contribute by sharing their point of views, similar or different experiences. All the sessions were recorded, while one person took notes of the session. While audio recordings was used as the main data for the analysis phase, we triangulated data using the other two sources of data (i.e. session notes and written reflections). It is recognized that in many research contexts working directly with recordings provide a better grasp and understanding of the phenomena under study [11]. The recordings were available to all members of the research team for re-analysis and clarification. Statements cited in the Results section are derived from these recordings.

The process of developing our individual reflections raised our awareness of our own biases, which are derived from our individual backgrounds. In particular, those derived from our 
cultural backgrounds and previous experiences. Therefore, we are transparent about the elements of such experiences that might have impacted our analysis. First, all the participants-researchers had previous academic experience in the U.S.; however, the length, intensity, and contexts of such experiences varied. One of us completed an undergraduate degree in the U.S. followed by a significant number of years of industry practice, while the other three obtained master's degrees in different universities and under different circumstances. One of us recognized that the opportunity available to get teaching certifications as a master student at a previous institution influenced significantly his confidence to communicate in verbal and written English previously to her Ph.D. endeavor.

We all recognize that these experiences might be significantly different than those of ESL students that are arriving to the U.S. as Ph.D. students for the first time. Even though we all were in that position as "first timers" before, some particular challenges pertain to our past, and not necessarily to our $\mathrm{Ph}$.D. experience. Therefore, our focus is more related to transitioning to a degree with the characteristics of a Ph.D. in engineering education despite of previous experiences. We find that such observations would be of value to ESL students independently of their length of stay in the U.S. In our findings section to keep our confidentiality and to make sure our examples remain unidentified, we decided to refer to ourselves (i.e., authors) by male pronouns while female pronouns would refer other people involved in our narratives.

\section{Culture}

\section{Findings}

The first inquiry subject that we examined was culture. In this topic, we decided to focus on the communication and interactional differences within cultures which influence our daily lives as graduate students. The themes that emerged from these discussions involved: (1) Mentorship Approaches, (2) Body Language Interpretation, and (3) Linguistic Efficacy. In our analysis, we decided to limit our cultural discussions to those related to academic environments. The discussions involved not only stating the challenges but how those challenges may differ from one participant to another considering that we come from three different ethnic backgrounds.

Mentorship approaches. When discussing how we come from different ethnic backgrounds, we concluded that self-interpretations and expectations among mentee-mentors and/or advisee/advisor were common challenges among us. We acknowledged that student's needs are different, and the amount and the type of coaching needed would differ from an individual to another. However, we believe that trust and expectations are important factors in generating a healthy relationship between faculty and students. One of the common threads that was discussed as a challenge is that some ESL students may have difficulties interpreting feedback. One of us stated:

"I have difficulty distinguishing faculty feedback and whether they are suggestions for improvements or "order to change"," 
The student noted that while both messages may convey students to improve, they are different in indicating whether the student is in good standing.

Body language interpretation. We have highlighted that the significance of body language differs from their own culture than in the United States. We indicated that we use body language as a means to gauge our relationship with our mentors, advisors, and peers and that the sense of inability to accurately interpret may create some uneasiness.

"Coming from my culture .. they are very expressive people .. I can tell emotional sense from the body language. Now I don't know where I am at .. because I no longer have that much of a body language to interpret from .. from the advisors that I worked with. I do feel that it is sort of a taboo to directly ask advisors where I am at .. as a Ph.D. student .. I feel that I should be beyond that point."

Linguistic efficacy. We defined linguistic efficacy as the confidence on our ability to communicate thoughts effectively. We noted that transitioning from other engineering fields to engineering education creates a sense of culture shock that may be amplified to ESL students. We, however, have noticed that this sense of uneasiness usually dissipates once ESL students realize that such difficulties are not inclusive to them and may be shared by native speakers of similar academic backgrounds. One of us mentioned how he restricted his communications with his peers and faculty due to his internal belief of the existence of a linguistic barrier.

\section{Language}

Language was the second inquiry subject that we examined. We agreed that language has mainly influenced us in three ways: (1) Interpersonal Communications, (2) Reading and (3) Writing. While reflecting on our experiences, we identified challenges that were common to all of us. Here we share these challenges and describe different ways we have overcome them. Besides, we provide suggestions to support others experiencing these challenges based on how we wished these challenges were supported by others and approaches we now believe we could have implemented for our benefit.

Interpersonal Communication. While reflecting on language, we all similarly believed that language has always been a self-confidence barrier to our communications with our native language speaker peers and faculty (advisors and course instructors). We considered communication in both verbal (in person) and written format (through emails/text messages). Challenges: We all experienced overwhelming moments of not being able to convey our messages clearly. This sometimes resulted in spending too much time explaining what we mean or writing long emails. In addition, the messages were sometimes misunderstood which caused frustrating moments for both senders and receivers of the message. While this is a common challenge among individuals who are not native language speakers, we believe that, if not 
supported properly, in the context of engineering education, language barrier can negatively influence one's progress.

One of us claimed that he started the program knowing that his English knowledge is sufficient for the program. However, he reflected on the experience that an email request he sent to a faculty member was not received well as a result of miscommunication which left him in trouble later. The faculty member later stated that she found the email disrespectful and she was not expecting to receive such emails from a $\mathrm{PhD}$ student.

We believe that this miscommunication may have been a result of the graduate student's language weaknesses and the cultural differences between him and the faculty. The graduate student of this story believes that since then his self-confidence in communicating with faculty members decreased and he has been afraid of having any request to any faculty members. As a result, he was not able to clearly express his needs and had missed some professional development opportunities he needed for his $\mathrm{PhD}$.

We believe that this moment could have turned into a learning opportunity for the graduate student if the faculty member addressed it differently. Based on other's similar experiences of miscommunication and misunderstanding, we believe that the graduate student would have benefitted if he was asked for clarifications regarding his message. It would have been a learning opportunity if he was told how to properly express his needs in the future. Similarly, one of us had reflected on how his self-confidence increased overtime as a result of his advisor and peers' support. The graduate student who started the program dealing with imposter syndrome given his communication skills, said:

"I was so lucky to have an advisor who acknowledged the effort I am putting in communicating with others in a different language than my native. I was also told by peers that they cannot imagine if they were doing their PhD in a different language than English. That was so encouraging for me to know that others know how hard English can be for me. So, I felt that it is okay if I make mistakes. I still spend a long time to write my emails, but it is fine if my emails are not perfect."

Also, he has stated that because his advisor has shown support in regard to his English, he can easily ask his advisor and even other peers to pronounce a word for him and/or to tell him how he can communicate a certain message to others.

The same graduate student as the previous example stated that his advisor's support provided him the comfort to ask her any questions regarding sending messages to different people. His advisor helped him write a message to his research participants for the first time. He was asked 
to write the message himself and then the advisor corrected the message to be formal and appropriate. Since then, he follows the same format to write his emails. Also, he confirmed:

"My advisor had helped me to respond to people or had also helped me in overcoming any misunderstandings I had from others."

The examples we provided here shed light on the importance of providing support to ESL students for their communication skills throughout their Engineering Education program. We can observe opposite experience of two students who started the program with different levels of comfort with English communication. One gained confidence given the support he received from the community, but the other one lost confidence given a negative moment he experienced.

Reading. Being successful in engineering education field requires lots of reading for both coursework and research work. Given our reflection, we realized that we all had struggled with reading materials for our coursework the first year of $\mathrm{PhD}$. Depending on what classes we were taking, we had to read up to 200 pages every week which was difficult even for our native language peers. We realized that we all had several stressful and frustrating moments for reading. A graduate student among us, said:

"Reading was the most stressful task for me in my first year. I had to read so many pages for each class every week. I literally cried many times as I couldn't do all my readings. I thought it's only me. But I never had the courage to ask my peers and cohorts, if reading is hard for them or not.... But one day, someone from my cohort told me that she couldn't read all his readings for the class and that is not his first time. I then realized that it's not only me! Reading is hard for all!"

Another graduate student reflected that:

"I don't remember any single time that I have done all my readings for my classes. But everyone seemed to know a lot in the class because they were talking about the topics.... Because I thought I am being compared with my other peers, I didn't find the need to even communicate my struggles."

The member of the team with the longer time in the US did not consider himself ESL when entering the PhD program, however he still thought that reading was very hard for him. He found talking about the readings and stating his own ideas very challenging in class.

In order to address these challenges, we all have utilized different resources and strategies to help us overcome reading challenges. Here we share the strategies that we found helpful.

1. ESL students need to know that their other peers may also struggle in reading. Therefore, we encourage faculty to discuss this possible challenge in their classes and provide suggestions and strategies for all students to get the most out of their readings. It 
is important that ESL students know that overtime they overcome those challenges as they learn and practice reading strategies. One author said:

"My advisor once asked me how I am doing with my classes, and I told them her about my readings. And she responded, "It is not only you who struggle, we hear this concern from many students. You need to start using strategies to read faster."

2. We all were under the impression that we need to read every single word of an article. However, we needed to know that skimming and speed-reading strategies are very helpful and necessary to learn to be successful in this program.

3. ESL students can always utilize resources like book or article reviews, summarizes and YouTube videos. We all agreed that each of these resources may hold some biases regarding the actual piece but reading them certainly helps graduate students to move forward with their classwork. While they do not replace becoming related with the original works, they certainly provide a baseline from which ideas can be build more strongly instead of relying on a dry first reading of dense academic contents.

4. ESL students can form reading groups. Three of us participated in reading groups in which readings of the week would be divided between the group members. The group would meet regularly every week to discuss the reading. The group members should read their own parts very carefully and be able to discuss them with others. The reading groups help students to master the readings that they have to read and learn about other readings as well.

5. ESL students are also encouraged to talk about their reading challenges to their cohort, their other peers and/or faculty to get possible suggestions and support.

6. Using tools that read aloud Pdf files is very helpful for those are slow readers. There are several applications that can be utilized.

Writing. To be successful in any Ph.D. program, graduate students required to be able to write extensively. In fields like engineering education that heavily relies on research, writing becomes more important as it is a form of communicating your outcomes. Writing was a struggle for all of us which we share some of the challenges below. In addition, we all benefited from different professional development experiences that we will share as suggestions for the engineering education, particularly ESL students.

We found that all of us were slow writers, if we wanted to be very careful about our writing. Being a slow writer, sometimes would have become very frustrating. One of us stated:

"Writing was hard and stressful. It takes a long time for me to write one paragraph. However, because I am compared to others, my effort was not recognized. One paragraph for me can take 3 times more, if not more, than others. I was told that I am not working hard, as I was not able to finish things on time." 
In addition to the time it would have taken us to write, some of us believed that the quality of our writing was not good enough for the courses we took. However, we have been able to overcome our writing challenges to some extent. We have listed the strategies that have worked for us as suggestions for other ESL students next:

1. Not all of the advisors and instructors are familiar with the challenges particular to ESL students. Therefore, we suggest ESL students to be clear with their advisors, that tell them that may not be a very strong writer, and they are willing to receive professional development on writing. This way advisors/instructors can provide necessary resources for the students to improve writing. They can also provide samples of assignments and statements that can help ESL students to understand how previous students have written them. One of us reflected on the relevance of this advisor/advisee communication as follows:

"I told my advisor that I am not good at writing, but I like to be able to write professionally. She reassured me that many students started the program struggling in writing, and they improved later. She edited my papers and I learned from her edits a lot. She also recommended me to take certain classes."

2. We suggest that ESL students gather information about the English courses and workshops that their school offers. The classes may be time consuming but can be very helpful. Many graduate programs and centers across at some institutions provide short professional development workshops that include writing and many other related topics like publishing a journal paper. We all benefited from the writing courses that were offered by our program.

3. Peer-review sessions are very helpful. Peers with different level of writing and expertise can help read each other's papers and provide effective feedback.

We all experienced peer-reviewing in different settings, classes, research groups or just two peers reviewing for one another. And we all strongly believe that we learn a lot from each other.

4. Many universities have writing centers that provide free tutors and writing groups that ESL student can benefit from, or even have general content that can be accessed as self-learning materials. Many of such resources can be accessed even across institutions. For example, the Purdue Online Writing Lab (https://owl.purdue.edu) offers a series materials that can support the advancement of any graduate students interested in advancing their skills.

\section{Discussion and Conclusion}

The objective of this paper was to share the experiences of ESL graduate students in an engineering education program. These experiences were described mainly around the challenges derived from the feelings of inadequacy in terms of cultural and language elements. We 
recognize that our view is particular to the evidence produced by our co-operative inquiry, therefore we do not intend to make any generalizations about the experiences of all ESL graduate students in the field. However, we find that our insights and suggestions might be useful for those that experience similar circumstances. In particular, we want to encourage ESL graduate students to become their own advocates in addressing their needs about improving their verbal and written communication. ESL students are more likely to be international students that besides the acculturation process in the academic space need to deal with adapting to living in the U.S. which is many times represent a radical change from their native cultures. Therefore, the sense of community we are disseminating here around a phenomenon that is usually experimented in isolation might be of value for ESL graduate students in similar paths.

We identified mentoring approaches as one of the main challenges derived from our limitations in communication as ESL graduate students. The advisor/advisee relationship has been identified before as one of the main factors affecting the persistence and success of graduate students in engineering [7-8], and in the experience of some of us, misunderstandings derived from both word choice and lack of cross-cultural awareness resulted in less than optimal relationships that somewhat undermined our self-confidence at some point of our doctoral journey. We invite ESL students to reflect constantly on which factors play a role into their negative experiences and keep a continuous improvement philosophy in which the lessons learned from these chapters can be used as sources of strength rather than infusing unbearable self-doubts. Similarly, we invite current faculty at graduate programs to seek higher awareness of cross-cultural differences that could be of value when dealing with ESL students. In particular, reflecting on how the limited communication skills might be undermining the efficiency in their verbal and written communication. Similarly, being aware of the resources that ESL students could use to strengthening these communication skills would add significant value to the build of trust in advisor/advisee relationships.

We found the exercise of this study to be of value for us as researchers and ESL professionals as an opportunity to develop our own identity as future academics. Our reflections made evident to us how relevant is to have authentic empathy for the experiences of students for minority groups. Some of us experienced what we perceived as higher levels of empathy from some faculty than others, such differences provide a lot of evidence on what we would have liked to do differently as mentees/advisees, as well as the things that we would like to mimic and what we would like to do differently as mentors/advisors. Finally, the list of suggestions offered here might be useful for similar graduate programs as a list of strategies that their ESL students can use to streamline their experiences and support their development. 


\section{References}

[1] S. Aguirre-Covarrubias, E. Arellano, and P. Espinoza, “'A pesar de todo' (Despite Everything): The Persistence of Latina Graduate Engineering Students at a Hispanic-Serving Institution," New Dir. High. Educ., vol. 2015, no. 172, pp. 49-57, Dec. 2015.

[2] V. Borum vborum@spelman. ed. and E. Walker, "What Makes the Difference? Black Women's Undergraduate and Graduate Experiences in Mathematics," J. Negro Educ., vol. 81, no. 4, pp. 366-378, Fall 2012.

[3] S. S. Canetto, C. D. Trott, J. J. Thomas, and C. A. Wynstra, "Making Sense of the Atmospheric Science Gender Gap: Do Female and Male Graduate Students Have Different Career Motives, Goals, and Challenges?," J. Geosci. Educ., vol. 60, pp. 408-416, Nov. 2012. [4] J. Jacobson, "Why Do So Many People Leave Graduate School Without a Ph.D.?," Chron. High. Educ. Wash., vol. 47, no. 38, p. A10, Jun. 2001.

[5] Council of Graduate Schools, "Ph.D. Completion Project."

[6] T. M. Evans, L. Bira, J. B. Gastelum, L. T. Weiss, and N. L. Vanderford, "Evidence for a mental health crisis in graduate education," Nat. Biotechnol., vol. 36, pp. 282-284, Mar. 2018.

[7] D. J. Satterfield, M. Tsugawa-Nieves, and A. N. Kirn, "WIP: Factors Affecting Graduate STEM Student Attrition Rates," p. 4.

[8] B. Miller, M. A. Tsugawa, J. N. Chestnut, H. Perkins, C. Cass, and A. Kirn, "The Influence of Perceived Identity Fit on Engineering Doctoral Student Motivation and Performance," presented at the 2017 ASEE Annual Conference \& Exposition, 2017.

[9] R. J. Aleong, M. H. Goldstein, J. D. O.-A. P.e, and H. E. Rodriguez-Simmonds, "Knowing Our Story: Framing a Cooperative Inquiry Project to Explore the Personal Growth of Graduate Students in Engineering Education," presented at the 2017 ASEE Annual Conference \& Exposition, 2017.

[10] J. Heron, Co-operative inquiry: research into the human condition. London; Thousand Oaks, Calif:: Sage Publications, 1996.

[11] S. Tessier, "From field notes, to transcripts, to tape recordings: Evolution or combination?" Intl. Journal of Qual. Methods, vol. 11, no. 4, p. 446-460, 2012. 